Article

\title{
The Longitudinal Pediatric Data Resource: Facilitating Longitu- dinal Collection of Health Information to Inform Clinical Care and Guide Newborn Screening Efforts
}

\section{Amy Brower ${ }^{1, *}$, Kee Chan, Michael Hartnett, Jennifer Taylor}

1 American College of Medical Genetics and Genomics (ACMG), 7101 Wisconsin Avenue Suite 1101, Bethesda, MD, 20814; E-Mail: abrower@ acmg.net; E-Mails: kchan@ @acmg.net; mhartnett@acmg.net; jtaylor@acmg.net.

* Author to whom correspondence should be addressed; E-Mail: abrower@acmg.net;

Tel.: +1-712-577-0666.

\begin{abstract}
The goal of newborn screening is to improve health outcomes by identifying and treating affected newborns. This manuscript provides an overview of a data tool to facilitate the longitudinal collection of health information on newborns diagnosed with a condition through NBS. The Newborn Screening Translational Research Network (NBSTRN) developed the Longitudinal Pediatric Data Resource (LPDR) to capture, store, analyze, visualize, and share genomic and phenotypic data over the lifespan of NBS identified newborns to facilitate understanding of genetic disease, and to assess the impact of early identification and treatment. NBSTRN developed a consensus-based process using clinical care experts to create, maintain, and evolve question and answer sets organized into common data elements (CDEs). The LPDR contains 24,172 core and disease-specific CDEs for 118 rare genetic diseases, and the CDEs are being made available through the NIH CDE Repository. The number of CDEs for each condition average of 2,200 with a range from 69 to 7,944. The LPDR is used by state NBS programs, clinical researchers, and community-based organizations. Case level, de-identified data sets are available for secondary research and data mining. The development of the LPDR for longitudinal data gathering, sharing, and analysis supports research and facilitates the translation of new discoveries into clinical practice.
\end{abstract}

(Maximum 189/200 words).

Keywords: newborn screening; research; long-term follow-up; NBSTRN; LPDR; RUSP. (3-10 keywords separated by semi colons)

\section{Introduction}

Each of the approximately 4 million babies born in the United States each year receives neonatal screening for over thirty-six rare genetic diseases. ${ }^{1}$ Newborns who screen positive undergo a series of 
confirmatory tests, and a subset ultimately gets a diagnosis. Screening and short-term follow-up occur within the state-based public health system while diagnosing, treating, and managing sick newborns occurs in pediatric care settings. This series of hand-offs from prenatal care to public health to clinical care creates a unique opportunity to capture crucial longitudinal health information for infants diagnosed through NBS. However, there is no national system to collect, analyze and share this information. ${ }^{2}$

Newborn screening is a system of interconnected activities that begin before a baby is born. This multi-stakeholder system's key components include prenatal education, neonatal screening, diagnosis, and treatment (Figure 1). Each year approximately 22,000 newborns are diagnosed with a condition through NBS, and the majority enter into life-long care management. ${ }^{3}$ However, the success of NBS has been measured by the number of newborns identified with a screened condition and not by health outcomes in diagnosed newborns. Our inability to quantify the net benefit of NBS by improved health outcomes may be a consequence of no national approach to longitudinal data collection, analysis, and dissemination. In the past ten years, a federal advisory committee, the Advisory Committee on Heritable Disorders in Newborns and Children (ACHDNC), has recognized the importance of long-term follow-up (LTFU) and

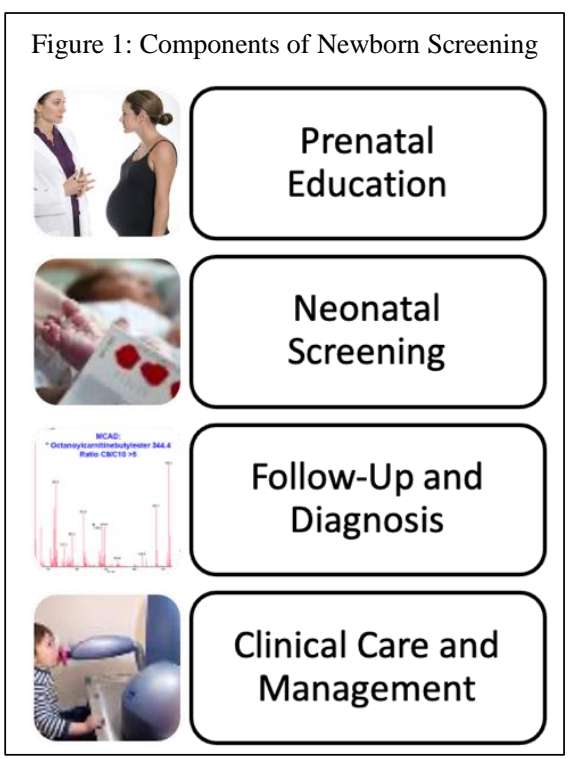
authored several publications defining the key features and components of NBS longitudinal data collection efforts, describing questions to be addressed, and identifying potential sources of data. ${ }^{4-6}$ ACHDNC defined the goal of LTFU as assuring the best possible outcome for individuals with disorders identified through NBS and identified key features and central components of LTFU (Figure 2).

The phrase "newborn screening long-term follow-up" applies both to the longitudinal treatment and management of diagnosed newborns as well as the longitudinal data collection of health information to inform assessments of outcomes. The Newborn Screening Saves Lives Reauthorization Act addressed LTFU as "follow-up activities, including those necessary to achieve rapid diagnosis in the short-term, and those that ascertain long-term management outcomes and appropriate access to related services." 7 Both activities are essential to advance NBS. The long-term clinical management of newborns diagnosed with a condition through newborn screening is necessary to ensure that we achieve the best possible outcomes for these infants. While the ability to capture clinical information early in the clinical course of a disease, even before clinical symptoms appear, advances disease understanding, helps to establish the efficacy of new treatments and management approaches, informs the community at large about the value of early identification and

Figure 2: ACHDNC Statement on LTFU

Assure the best possible outcome for individuals with disorders identified through newborn screening

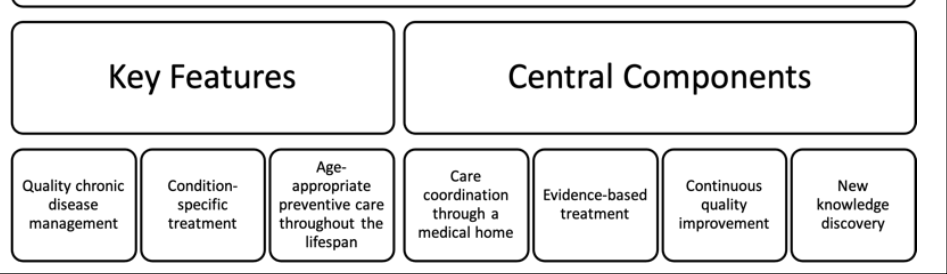
treatment through newborn screening, and identifies areas for improvement in disease management throughout the lifespan. Also, longitudinal data collected on children diagnosed through populationbased screening offers significant potential to advance scientific and clinical understanding of NBS conditions and document the life-saving benefit of NBS. It enables individual and group level analysis 
from a data set with unbiased ascertainment. Longitudinal Data also has the capability to capture information on family experience, improving the capacity at which clinical experts can care for their patients and their families. In this paper, we describe efforts to facilitate the longitudinal capture and dissemination of health information on newborns diagnosed with a condition through NBS.

\section{Materials and Methods}

\subsection{Newborn Screening Translational Research Network}

The Eunice Kennedy Shriver National Institute of Child Health and Human Development (NICHD) Hunter Kelly Newborn Screening Research Program was created to support investigations and newborn screening innovations. ${ }^{8}$ Recent efforts have explored the use of genomics in the neonatal period, conducted prospective pilots of conditions that are candidates for nationwide screening to evaluate the clinical benefit, and developed novel screening technologies for candidate conditions. The American College of Medical Genetics and Genomics (ACMG) plays a vital role in these groundbreaking efforts by leading the NICHD funded Newborn Screening Translational Research Network. NBSTRN is a key component of the Hunter Kelly Newborn Screening Research Program and began as an effort to engage a variety of stakeholders across the NBS system. ${ }^{9}$ NBSTRN has now matured into a dynamic and committed network comprised of researchers, healthcare professionals, state NBS programs, families, and advocacy groups. The NBSTRN team develops tools to facilitate the discovery and validation of novel technologies to screen and diagnose disease, pilot new technologies and treatments, describe the ethical, legal, and social implications of NBS research, and collect longitudinal health and genomic data.

NBS in the US is a multi-component, multi-stakeholder system of prenatal education, hospital and state-based public health laboratory screening, clinician and state-based laboratory confirmation and diagnosis, clinical treatment and management, and health outcome analysis. The NBSTRN data tools, resources, and expertise are designed to facilitate all stakeholders' efforts and leverages each component of NBS to advance research. As a translational research network, NBSTRN was conceptualized to develop and share resources and infrastructure to support NBS researchers. The collective research infrastructure provides mechanisms for an unbiased understanding of rare genetic conditions across the lifespan, population-based pilot testing of new NBS tests, new technology development and application, and new therapeutics development. This research infrastructure also strengthens and enables the expansion of NBS programs in a more systematic fashion.

\subsection{Development of Common Data Elements (CDEs)}

ACMG also operates the National Coordinating Center (NCC) for the Regional Genetics Networks (RGNs). ${ }^{10}$ In 2015, two representatives from each of the seven RGNs formed a joint committee with an expert NBSTRN workgroup called the Clinical Integration Group (CIG). The joint committee of experts developed sets of questions and answer choices, called common data elements (CDEs), for NBS conditions that are part of the Recommended Uniform Screening Panel (RUSP). ${ }^{11}$ In newborn screening, the use, and development of CDEs are focused on facilitating data collection, standardization, sharing, aggregation, analysis, and dissemination. Combining data sets is especially important in newborn screening because most conditions are rare, and accumulating enough subjects to have 
statistical power is often a barrier to understanding health outcomes and the benefits of early identification and treatment.

The goal was to create a catalog of CDEs to enable investigators and public health teams to systematically collect, analyze, and share data across the NBS community. The development of the CDEs was guided by ACHDNC's publications and Follow-Up and Treatment Subcommittee discussions. ${ }^{12}$ The CDEs were designed to be applicable for both research and public health and contained core and disease-specific CDEs. The NBS CDE sets' potential uses include natural history studies, hypothesis-driven efforts, surveillance, outcomes, quality assurance and improvement, and national benchmarks. A clinician may use the NBS CDE sets to describe the clinical course of the NBS identified condition in asymptomatic patients to facilitate a new understanding of the disease. At the same time, another may explore the relationship between genomic variants characterized at birth and later outcomes like lung function in cystic fibrosis. A public health partner may use the NBS CDE sets to describe the relationship between service delivery and treatment methods to define optimal follow-up care plans for children with an NBS condition.

NBSTRN was charged with creating a data tool using the resulting consensus-based, standardized CDEs, organizing the CDEs into case report forms, and making the forms available through a secure online portal facilitating data collection and management. NBSTRN also worked with subject matter experts to develop CDE sets for conditions that are candidates for newborn screening. CDE dissemination was accomplished through a collaboration with the National Institutes of Health (NIH) National Library of Medicine (NLM) and NBSTRN via the NIH CDE Repository (Repository). ${ }^{13}$ Currently, this repository catalogs 26,518 elements across 16 classifications, with multiple NIH Institutes and efforts being represented. The Repository allows researchers to build data collection instruments from shared CDEs and also contribute generated data elements. To foster the use of standardized CDEs, NBSTRN deposits the question and answer sets in the NICHD module of the Repository. This repository has been designed to provide access to structured human and machinereadable definitions of data elements that have been recommended or required by NIH Institutes and Centers and other organizations for use in research and other purposes.

\subsection{Longitudinal Pediatric Data Resource}

The neonatal screening of newborns each year leads to the diagnosis of infants with a genetic condition that requires referral to clinical care and, in most cases, lifelong management. This unselected cohort of newborns reflects our nation's racial, geographic, economic, and educational diversity. This may be the perfect cohort to help advance disease understanding because although every newborn receives essentially the same screen, other factors vary, including treatment choice and disease course. Also, many of the screened conditions have comorbidities, including intellectual disabilities. These children receive various interventions that could be tracked and analyzed to identify critical periods of development and intervention. Because the NBS system in the United States effectively screens over 99\% of newborns, it can provide a unique platform for understanding rare diseases and lifelong outcomes. The process of neonatal screening followed by a coordinated transition to clinical care facilitates collecting health information beginning just hours after birth. And because the majority of 
NBS conditions require life-long care and management, we have the opportunity to conduct prospective, longitudinal natural history studies on a population basis with unbiased ascertainment.

Over the last decade, NBSTRN has developed a data tool, the Longitudinal Pediatric Data Resource (LPDR), to support several landmark natural history studies that have contributed to a better understanding of the etiology, pathophysiology, and phenotypic heterogeneity of NBS conditions and provided an assessment of health outcomes. ${ }^{14}$ The LPDR is a suite of tools to collect, analyze, visualize, store, and share genomic and phenotypic data. The LPDR is housed within a FISMA Moderate cloud environment, is available for use by all NBSTRN stakeholders, and is designed to share both deidentified, case-level and aggregate data sets and foster secondary use of accumulated data. Data stored in the LPDR is available to the research and NBS community, and the use of de-identified, case-level data requires registration as an NBSTRN user. Depositing case-level data into the LPDR and requesting the use of complete data sets that include personal health information (PHI) involves the execution of a Data Sharing Agreement (DSA). The LPDR also facilitates data sharing and data standardization with $3^{\text {rd }}$ party databases, including the NIH National Center for Biotechnology Information (NCBI) database of Genotypes and Phenotype (dbGaP) and the NLM NIH Code Repository (https://cde.nlm.nih.gov). The LPDR provides data dictionaries to create electronic data entry forms and features de-identified, case-level data sets for data mining. The secondary use of accrued LPDR data may help to 1) establish the efficacy of new treatments and management approaches, 2) inform the community about the value of early identification and treatment through newborn screening, and 3) identify areas for improvement in disease management throughout the lifespan.

The LPDR is accessible through the NBSTRN website at nbstrn.org and operates in a cloud environment that utilizes the NIH Science and Technology Research Infrastructure for Discovery, Experimentation, and Sustainability (STRIDES) Initiative and Amazon Web Services (AWS). ${ }^{15}$ The NBSTRN team follows federal policies for information security protections outlined under the Federal Information Security Management Act (FISMA) of 2002 (Public Law 107-347). ${ }^{16}$ These policies outline requirements for developing and operating information technology (IT) systems based on data type, data use, and risk impact. There are three security categories-low, moderate, or high. ACMG received authority to operate (ATO) the NBSTRN website, tools, and resources at the moderate level, and this enables the collection, storage, and use of personal health information (PHI). ACMG submits a quarterly review of the system's security to NICHD, and this provides the NBSTRN network with an approved infrastructure to facilitate the protected and secure collection, analysis, and dissemination of NBS research data.

\section{Results}

\subsection{CDE Sets}

A total of 24,172 core and disease-specific CDEs have been developed for use in longitudinal health information data collection efforts for 118 conditions that are part of, or candidates for, nationwide NBS. The joint committee created 15,685 CDEs for 46 RUSP conditions. The NBSTRN developed 8,487 additional disease-specific CDEs for 72 diseases for use by researchers conducting natural history studies or pilots of conditions recently added to, or candidates for, the RUSP (Appendix). The CDEs were organized into data dictionaries and electronic case report forms (eCRFs) that incorporated the CDEs 
into dropdown menus using REDCap. ${ }^{17}$ The CDEs were made available to the NLM for dissemination via the NIH CDE Repository, NICHD Module, and integration into EHR standards for the US and the NBS Coding and Terminology Guide. ${ }^{18}$ A data almanac was created that contained semantic definitions, annotations, and standardization to improve the data collected by each investigator. 3745 of the 15,685 (24\%) of the CDEs currently have definitions and, where applicable, annotated with Logical Observation Identifiers Names and Codes (LOINC), Systematized Nomenclature of Medicine-Clinical Terms (SNOMED CT), and the International Classification of Diseases, Ninth or Tenth Revision (ICD-9 or 10) codes. $^{18}$ Disease-specific and study-specific $\mathrm{CDE}$ sets are available online at https://nbstrn.org/tools/lpdr. CDE sets are organized into REDCap formatted data dictionaries and text files. The LPDR enables users to adopt the consensus-based CDEs, generate case report forms, and suggest additional CDEs for new conditions, technologies, and treatments.

\subsection{LPDR: Phenotypic and Genomic Data}

Since its launch in 2013, the LPDR has been utilized by several research teams, either conducting longitudinal studies of both RUSP and candidate conditions, exploring the use of genome sequencing in the newborn period, and executing NBS pilots of candidate conditions. The LPDR has 312 registered users, 151 individuals approved to enter or upload data, 23 established longitudinal projects, $\sim 12$ Million data points, and 8,842 subjects with an average of 3.62 data collection time points per subject. The LPDR has data displays that describe the types of data and populations available for secondary use. Our data governance and data sharing policies provide qualified researchers access to these data sets. Case-level, de-identified data sets from 8 projects are featured within the LPDR and are available for secondary use by the research community. Data dashboards for the eight featured projects highlight critical aspects of the accumulated data and population. Thirteen active and completed projects provide a representative sample of the type of efforts that utilize the LPDR (Table 1). 
Table 1: Summary of Thirteen Active and Completed Projects in the LPDR

\begin{tabular}{|c|c|c|c|c|c|c|c|c|}
\hline Project & Condition(s) & Population & CDEs & Subjects & $\begin{array}{l}\text { Max } \\
\text { Data } \\
\text { Entry } \\
\text { Time } \\
\text { Points }\end{array}$ & User Type & $\begin{array}{c}\text { Data } \\
\text { Available?* } \\
\text { (Yes/No) }\end{array}$ & Status \\
\hline $\begin{array}{l}\text { Sickle Cell } \\
\text { Disease Newborn } \\
\text { Screening } \\
\text { Follow-Up } \\
\end{array}$ & Sickle Cell Disease & $\begin{array}{l}\text { Diagnosed } \\
\text { Cases }\end{array}$ & 57 & 8,346 & 9 & $\begin{array}{l}\text { Community- } \\
\text { Based } \\
\text { Organization } \\
\text { (CBO) } \\
\end{array}$ & Yes & Active \\
\hline $\begin{array}{l}\text { Inborn Errors in } \\
\text { Metabolism } \\
\text { Consortium } \\
\text { (IBEMC) } \\
\end{array}$ & 43 RUSP Conditions** & $\begin{array}{l}\text { Diagnosed } \\
\text { Cases }\end{array}$ & 2,649 & 2,078 & 30 & Clinician & Yes & Completed \\
\hline $\begin{array}{l}\text { Project CURE } \\
\text { Spinal Muscular } \\
\text { Atrophy (SMA) } \\
\end{array}$ & SMA & $\begin{array}{l}\text { Diagnosed } \\
\text { Cases }\end{array}$ & 4,337 & 701 & 5 & Clinician & Yes & Active \\
\hline SPOT SMA & SMA & Newborns & 4,759 & 434 & 4 & Clinician & Yes & Active \\
\hline Krabbe Registry & Krabbe & $\begin{array}{l}\text { Diagnosed } \\
\text { Newborns }\end{array}$ & 187 & 196 & 1 & $\begin{array}{l}\text { Clinician and } \\
\text { Parent }\end{array}$ & Yes & Completed \\
\hline Rady NSIGHT2 & Variety & $\begin{array}{l}\text { Neonatal } \\
\text { Intensive } \\
\text { Care Unit } \\
\text { (NICU) }\end{array}$ & 45 & 214 & 2 & Clinician & Yes & Completed \\
\hline BabySeq & Variety & $\begin{array}{l}\text { NICU; } \\
\text { Healthy at } \\
\text { Birth }\end{array}$ & 30 & 159 & 2 & Clinician & Yes & Completed \\
\hline NC NEXUS & RUSP $* * *$ & Newborns & 180 & 200 & 2 & Clinician & Yes & Completed \\
\hline NY State LTFU & $\begin{array}{l}\text { X-linked } \\
\text { Adrenoleukodystrophy, Severe } \\
\text { Combined Immune } \\
\text { Deficiency, Fatty Acid } \\
\text { Oxidation Disorders, Krabbe }\end{array}$ & Newborns & 202 & 10 & 5 & $\begin{array}{l}\text { State NBS } \\
\text { Program }\end{array}$ & No & Active \\
\hline $\begin{array}{l}\text { Duchenne NBS } \\
\text { Pilot }\end{array}$ & $\begin{array}{l}\text { Duchenne Muscular } \\
\text { Dystrophy }\end{array}$ & Newborns & 624 & 17 & 6 & $\begin{array}{l}\text { Clinician and } \\
\text { State NBS } \\
\text { Program } \\
\end{array}$ & No & Active \\
\hline $\begin{array}{l}\text { eXtraordinarY } \\
\text { Babies Study }\end{array}$ & Chromosome Aneuploidy & Newborns & 320 & 200 & 5 & Clinician & No & Active \\
\hline $\begin{array}{l}\text { CPT1A Arctic } \\
\text { Variant }\end{array}$ & CPT1A Arctic Variant & $\begin{array}{l}\text { Newborn } \\
\text { and Children }\end{array}$ & 69 & TBD & 4 & Clinician & No & Active \\
\hline $\begin{array}{l}\text { Oregon Health \& } \\
\text { Science } \\
\text { University }\end{array}$ & RUSP**** & Newborns & 894 & TBD & 2 & Clinician & No & Active \\
\hline
\end{tabular}

*Data availability subject to approval from the data submitter/owner.

**2-Methyl-3-hydroxybutyric aciduria, 2-Methylbutyrylglycinuria, 3-Methylcrotonyl-CoA carboxylase deficiency, 3-Methylglutaconic aciduria, Argininosuccinic aciduria, Disorders of biopterin biosynthesis, Disorders of biopterin regeneration, Biotinidase deficiency, beta-Ketothiolase deficiency, Carnitine-acylcarnitine translocase deficiency, Methylmalonic acidemia (Cobalamin disorders), Methylmalonic acidemia (Cobalamin disorders), Primary Congenital Hypothyroidism, Citrullinemia type I, Citrullinemia type II, Carnitine palmitoyltransferase I deficiency, Carnitine palmitoyltransferase II deficiency, Carnitine uptake defect, Glutaric acidemia type I, Glutaric acidemia type II, Galactoepimerase deficiency (uridine diphosphate galactose 4-epimerase deficiency), Galactokinase deficiency, Classical galactosemia (galactose-1-phosphate uridyltransferase deficiency), Hyperphenylalaninemia (variant, benign), Homocystinuria, 3Hydroxy-3-methylglutaric aciduria, Isobutyrylglycinuria, Isovaleric acidemia, Malonic acidemia, Medium-chain acyl-CoA dehydrogenase deficiency, Multiple carboxylase deficiency, Hypermethioninemia, Maple syrup urine disease, Methylmalonic acidemia (methylmalonyl-CoA mutase), Phenylketonuria, Proionic acidemia, Short-chain acyl-CoA dehydrogenase deficiency, Trifunctional protein deficiency, Tyrosinemia type I, Tyrosinemia type II, Tyrosinemia type III, Very long-chain acyl-CoA dehydrogenase deficiency

***https://www.hrsa.gov/advisory-committees/heritable-disorders/rusp/index.html

\section{Discussion}

Longitudinal care management of newborn screen identified individuals coupled with longitudinal capture of health information are vital cornerstones of a successful NBS system in the United States. The NBSTRN led an effort to develop the LPDR, a suite of tools and resources to facilitate longitudinal data capture using question and answer sets developed by clinical experts. This effort resulted in the identification of key data elements for long-term follow-up (LTFU) efforts conducted by state NBS programs. These data elements could also be used by researchers, advocates, policy makers, and clinicians to evaluate the health outcomes of newborns identified by NBS. The LPDR has been utilized in a variety of efforts, including a ten-year effort to collect, analyze and disseminate health information on individuals with one of 42 RUSP conditions in 30 clinical sites located in 22 states, multistate pilots of 4 conditions collectively screening over $1.2 \mathrm{M}$ births, use of genome sequencing in four 
cohorts of newborns including neonatal intensive care unit and healthy, studies expanding the diagnostic window of NBS both beyond and before the neonatal period, and efforts to consolidate disparate patient registries into a single data dictionary that supports pilots. As the data sets contained in the LPDR grow, the NBS community can utilize this information to inform the care and management of newborns identified with a condition through NBS, guide policy and funding decisions, assess the benefit of NBS, and further our understanding of these rare genetic conditions.

To facilitate the utilization and application of LDPR, NBSTRN has displayed a set of LPDR studies and data visualization on the website www.nbstrn.org (see Figure \#). By becoming a member of NBSTRN, patrons can have access to the de-identified case-level data, conduct secondary data analysis and explore additional research questions such as how often do individuals with metabolic diseases have hearing loss.

Figure 3. LPDR Projects Available for Secondary Use

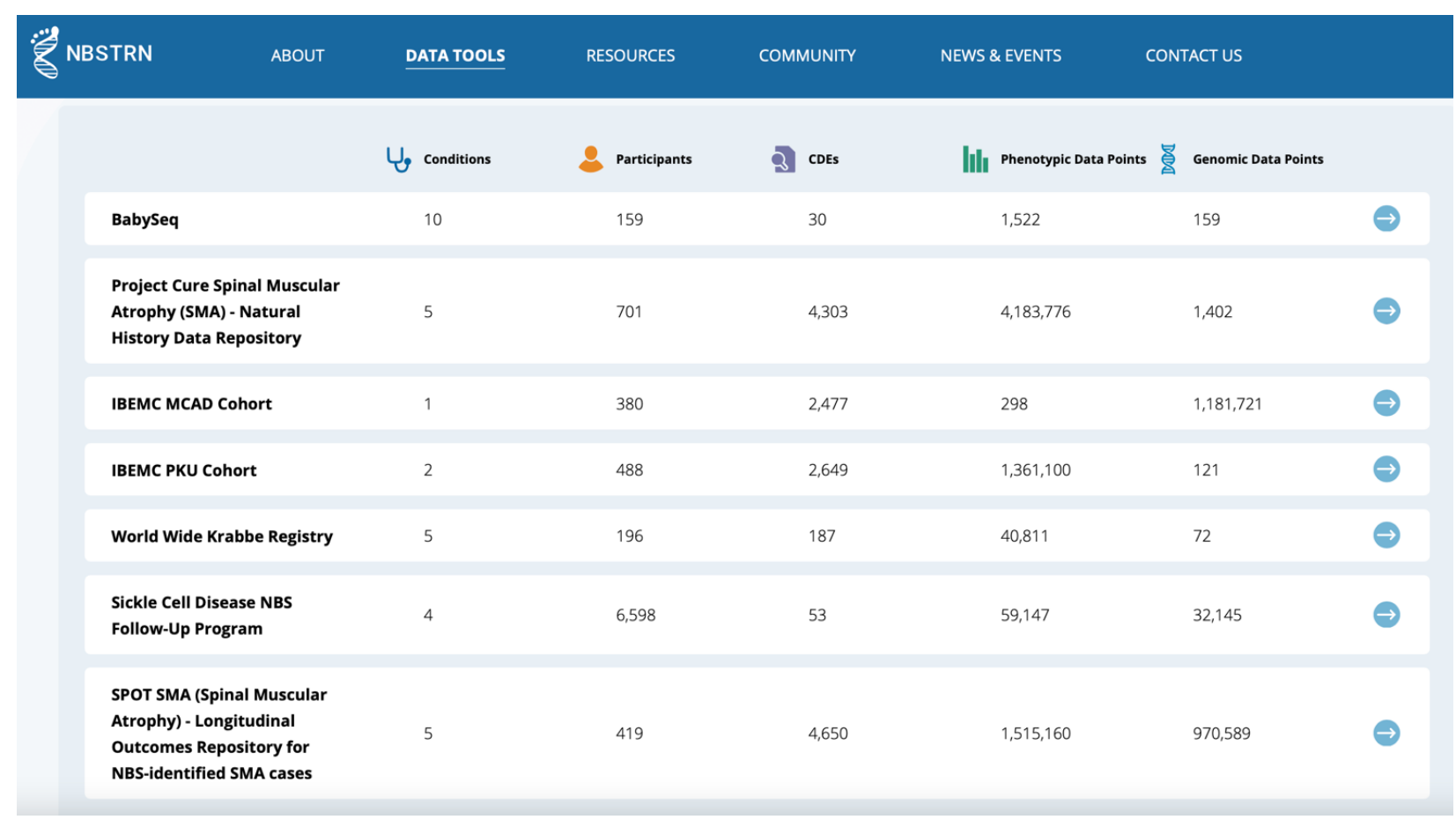


Figure 4. LPDR Data Visualization
Ч $\stackrel{\mathbf{5}}{\text { Conditions }}$
- 419
8. $\begin{aligned} & \mathbf{4} 650 \\ & \text { CDES }\end{aligned}$
1,515,160
870,589
Genomic Data Points

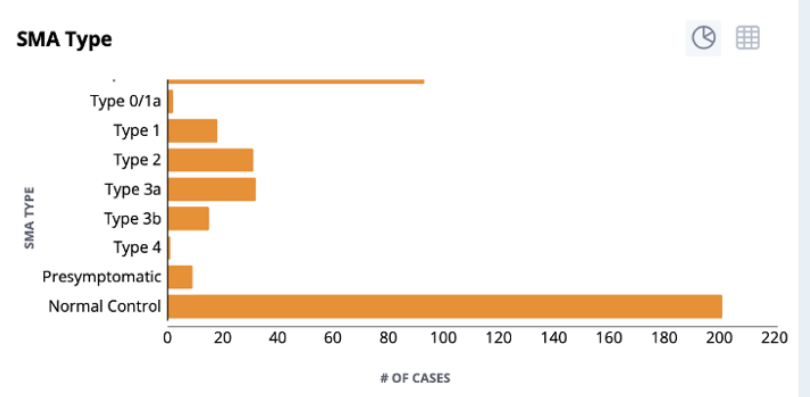

SMN2 Copy Number

(Q) 圇

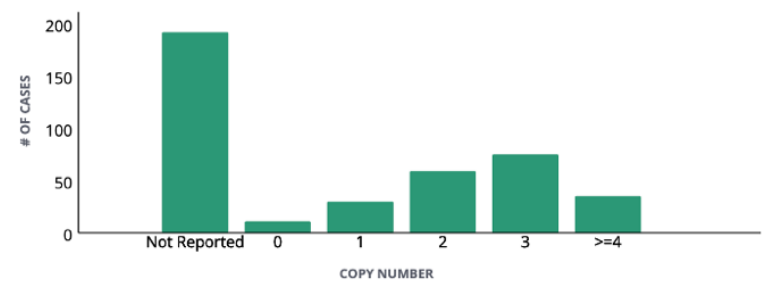

Treatment Type

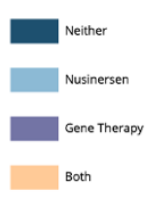

(2) 用

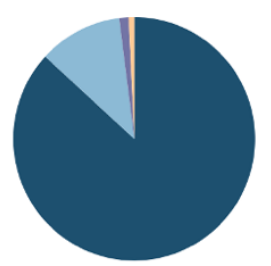

Gender

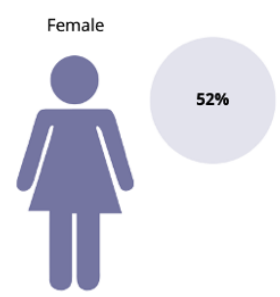

(b) 用

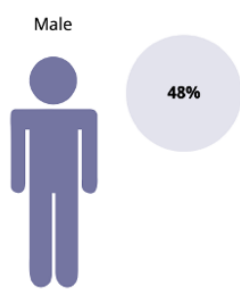


"The authors declare no conflict of interest."

\section{References and Notes}

1. Advisory Committee on Heritable Disorders in Newborns and Children - Recommended Uniform Screening Panel. Available online: https://www.hrsa.gov/advisorycommittees/mchbadvisory/heritabledisorders/recommendedpanel/in dex.html. 12 April 2021

2. Puryear, MA.; Brower, AM. Long-term follow-up in newborn screening: a systems approach for improving health outcomes. Genet Med, 2010, 12, S256-260.

3. Brower, AM. Counting Conditions.

4. Kemper, AR, et al. Long-term follow-up after diagnosis resulting from newborn screening: statement of the US Secretary of Health and Human Services' Advisory Committee on Heritable Disorders and Genetic Diseases in Newborns and Children. Genet Med, 2008, 10(4):259061.

5. Hinton, $\mathrm{CF}$, et al. What questions should newborn screening long-term follow-up be able to answer? A statement of the US Secretary for Health and Human Services' Advisory Committee on Heritable Disorders in Newborns and Children. Genet Med, 2011, 13(1):861-5.

6. Hinton, CF, et al. A framework for assessing outcomes from newborn screening: on the road to measuring its promise. Mol Genet Metab, 2016, 118(4):221-229.

7. Senate of the United States. H.R.1281 -Newborn Screening Saves Lives Reauthorization Act of 2014. Available online: https://www.congress.gov/bill/113th-congress/house-bill/1281. 12 April 2021.

8. Alexander, D, Hansen JW. NICHD research initiative in newborn screening. Ment Retard Dev Disabil Res Rev, 2006, 12:229-315.

9. Puryear M, et al. Foundation of the Newborn Screening Translational Research Network and its tools for research. Genet Med, 2019, 21(6):1271-1279.

10. Kaye, C, et al. Regional models of genetic services in the United States. Genet Med, 2020, 22(2):381-388.

11. Brower, AM. Long-Term Follow-Up in Newborn Screening: An Update on ACMG's Work on the NBSTRN and NCC. Available online: https://www.hrsa.gov/sites/default/files/hrsa/advisorycommittees/heritable-disorders/meetings/Heritable\%20Disorders\%202004-2015/2015/May\%201112,\%202015/brower.pdf. 13 April 2021.

12. The Role of Quality Measures to Promote Long-Term Follow-up of Children Identified by Newborn Screening Programs. Available online:

https://www.hrsa.gov/sites/default/files/hrsa/advisory-committees/heritable-disorders/reportsrecommendations/reports/role-of-quality-measures-in-nbs-sept2018-508c.pdf. 13 April 2021.

13. NIH CDE Repository. Available online: https://cde.nlm.nih.gov/cde/search?selectedOrg=NICHD. 12 April 2021.

14. Longitudinal Pediatric Data Resource (LPDR), Available online: https://nbstrn.org/tools/lpdr. 12 April 2021.

15. NIH Science and Technology Research Infrastructure for Discovery, Experimentation, and Sustainability (STRIDES) Initiativehttps://datascience.nih.gov/strides. 13 April 2021.

16. Public Law 107-347. Available online: https://www.congress.gov/107/plaws/publ347/PLAW107pub1347.pdf. 
17. Obeid, JS, et al. Procurement of shared data instruments for Research Electronic Data Capture (REDCap). 2013, J Biomed Info, 2013, Vol 46:2:259-265.

18. Newborn Screening Coding and Terminology Guide. Available online: https://hncbc.nlm.nih.gov/newbornscreeningcodes/. 13 April 2021.

Appendix: List of 118 Conditions with CDE Sets

,2-Methyl-3-hydroxybutyric aciduria, 2-Methylbutyrylglycinuria, 2,4 Dienoyl-CoA reductase deficiency, 3-Hydroxy-3-Methyglutaric Aciduria, 3-Methylcrotonyl-CoA Carboxylase Deficiency, 3Methylglutaconic aciduria, 3-Phosphoglycerate dehydrogenase deficiency, Adenine phosphoribosyltransferase deficiency, Alpha-N-acetylglucosaminidase deficiency, Arginine:Glycine Amidinotransferase (AGAT) Deficiency, Argininemia, Argininosuccinic Aciduria, Arylsulfatase B deficiency (Maroteaux-Lamy syndrome) (MPS VI), Benign hyperphenylalaninemia, Beta-glucuronidase deficiency (Sly syndrome) (MPS VII), Beta-Ketothiolase Deficiency, Biopterin defect in cofactor biosynthesis, Biopterin defect in cofactor regeneration, Biotinidase Deficiency, Branched-chain ketoacid dehydrogenase kinase, Brown Vialetto Van Laere Syndrome (Riboflavin transporter), Carbamoyl Phosphate Synthase (CPS) Deficiency, Carbonic anhydrase VA deficiency, Carnitine acylcarnitine translocase deficiency, Carnitine palmitoyltransferase type I deficiency, Carnitine palmitoyltransferase type II deficiency, Carnitine Uptake Defect/Carnitine Transport Defect, Cerebrotendineous xanthomatosis, Citrullinemia, Type I, Citrullinemia, type II, Cobalamin C,D Deficiency, Congenital adrenal hyperplasia, Congenital Cytomegalovirus infection, Congenital Human immunodeficiency virus infection, Congenital Toxoplasmosis infection, Copper transport disease (Menkes disease), Creatine Transporter (CRTR) Deficiency, Critical Congenital Heart Disease, Cystic Fibrosis, Di George syndrome, Duchenne muscular dystrophy, Ethylmalonic encephalopathy, Fabry Disease, Familia hypercholesterolemia, Formiminoglutamic acidemia, Fragile $\mathrm{X}$ syndrome, Friedreich ataxia, Galactoepimerase deficiency, Galactokinase deficiency, Galactosamine 6-sulfatase deficiency (Morquio), Galactosemia, Galactosialidosis, GAMT deficiency, Gaucher disease, Glucose-6-phosphate dehydrogenase deficiency, Glutaric Acidemia Type I, Glutaric acidemia type II, Hearing Loss, Hemoglobin H (Bart), Heparan sulfate sulfatase deficiency (San Filippo), Hepatolenticular degeneration (Wilson disease), Holocarboxylase Synthase Deficiency, Homocystinuria, Hypermethioninemia, Hyperornithinemia with Gyrate Deficiency, Hyperornithinemia-hyperammonemia-homocitrullinemia syndrome, Iduronate 2-sulfatase deficiency (MPSII), Isobutyrylglycinuria, Isovaleric Acidemia, Kernicterus, Krabbe Disease, Long-chain L-3 Hydroxyacyl-CoA Dehydrogenase Deficiency, Lysosomal acid lipase deficiency (Wolman disease), Malonic acidemia, Maple Syrup Urine Disease, Medium-chain Acyl-CoA Dehydrogenase Deficiency, Medium-chain ketoacyl-CoA thiolase deficiency, Medium/short-chain L-3-hydroxyacyl- CoA dehydrogenase deficiency, Metachromatic leukodystrophy, Methylmalonic Acidemia (Cobalamin disorders), Methylmalonic Acidemia (methylmalonyl-CoA mutase), Methylmalonic acidemia with homocystinuria, Molybdenum cofactor deficiency, Mucopolysaccharidosis II, Mucopolysaccharidosis Type 1, N-Acetylglutamate Synthase (NAGS) Deficiency, N(5,10)-Methylene tetrahydrofolate reductase, Niemann Pick Disease, Nonketotic Hyperglycinemia, Ornithine Transcarbamylase (OTC) Deficiency, Phenylketonuria, Pompe, Primary Congenital Hypothyroidism, Prolinemia Type I/Type II, Propionic Acidemia, Proximal urea cycle defects, Pyridoxine Responsive Epilepsy, Pyroglutamic acidemia, Pyruvate carboxylase deficiency, Pyruvate dehydrogenase lipoic acid synthetase, S, Beta-Thalassemia, S,C Disease, S,S Disease (Sickle Cell Anemia), Severe Combined Immunodeficiencies, Short-chain acyl-CoA dehydrogenase deficiency, Smith Lemli Opitz syndrome, Spinal muscular atrophy, Spinal Muscular Atrophy due to homozygous deletion of exon 7 in SMN1, T-cell related lymphocyte deficiencies, Trifunctional Protein Deficiency, Tripeptidyl peptidase 1 deficiency (Neuronal Ceroid Lipofuscinosis 2), Tyrosinemia, Type I, Tyrosinemia, type II, Tyrosinemia, type III, Various other hemoglobinopathies, Very Long-chain AcylCoA Dehydrogenase Deficiency, X-linked Adrenoleukodystrophy, Zellweger Syndrome. 
(C) 2014 by the authors; licensee MDPI, Basel, Switzerland. This article is an open access article distributed under the terms and conditions of the Creative Commons Attribution license (http://creativecommons.org/licenses/by/4.0/). 